\title{
From the Guest Editors
}

This issue of Oceanography focuses on seamounts, submerged isolated mountains in the sea that are hosts to complex and intertwined, dynamic physical, chemical, and biological systems. More than $99 \%$ of all seamounts on Earth remain unexplored, but studies of a very small subset so far show that they have and continue to provide exciting research opportunities across many science disciplines. Magmatic processes that originate deep in Earth's mantle build volcanoes-seamountsthat can reach heights of up to $9 \mathrm{~km}$ above the seafloor. Volcanically active seamounts develop hydrothermal systems that vent hot, metal-rich, reducing, and often acidic fluids and that result in a considerable chemical exchange of elements and volatiles with seawater. In addition, many seamounts provide major fluid pathways between the underlying oceanic crust and the ocean, bypassing the impermeable sediment layer covering most of the oceanic crust. Seamounts also interfere with ocean currents to yield local circulation patterns that help mix the ocean, resuspend sediments, and perhaps locally trap or enhance the abundance of plankton.

All these physical, chemical, hydrological, and oceanographic processes at seamounts provide the basis for development of some of the most diverse and richest biological "hotspots" in the ocean. Microbes benefit from the wide availability of reductive energy and metals from hydrothermal systems around seamounts or from nutrients brought to these volcanic structures by ocean currents and deep-water upwelling. Microbial communities at seamounts represent the lowest trophic level of a complex seamount food web that is prominently capped with some of the most productive fisheries in the ocean. Cumulatively, all seamounts together form a vast biome that rivals and maybe exceeds one of mountain zones on land. They are likely to play a key role in the ocean ecosystem, but they also remain the leastexplored major biome on Earth. Overfishing and seafloor habitat destruction associated with fish trawling gear makes seamounts the targets of conservation scientists and creates the need for international advocacy and political cooperation to regulate seamount fisheries.

Much of today's seamount science is done in isolation from other disciplines, and seamount chemistry, physics, geology, hydrology, oceanography, biology, and fisheries are pursued largely independently. Yet, it is clear that seamount researchers appreciate and are aware of the cross-disciplinary connectivity and feedbacks at these mountains in the sea. For example, the biology and fisheries of seamounts depend largely on fluid flow and nutrient supply that are related to chemical fluxes from the seamount and from the surrounding ocean. Chemical fluxes can support microbial growth, but the microbes themselves can provide a feedback mechanism through their impact on chemical fluxes by dissolving seamount rocks or precipitating biominerals. Much can be gained from further cooperation and mutual understanding between disciplines to identify the most promising collaborative opportunities to improve our understanding of the relationships among the physical, chemical, and biological systems at seamounts.

This issue of Oceanography offers a broad perspective on seamount research to raise awareness of the diversity of seamount research and to promote collaboration among seamount scientists. It is based on a recent workshop, Seamounts '09, organized in March 2009 at Scripps Institution of Oceanography (http://earthref.org/events/SBN/2009/ for program and downloadable keynote presentations) by the Seamount Biogeoscience Network (SBN; http://earthref.org/ $\mathrm{SBN})$. Contributions to this issue include a series of reviews and original papers focused on key issues in seamount research and cover a wide spectrum of seamount science disciplines. In addition, the reader can find short "spotlights" that feature specific seamounts that were studied in depth. This issue also makes use 


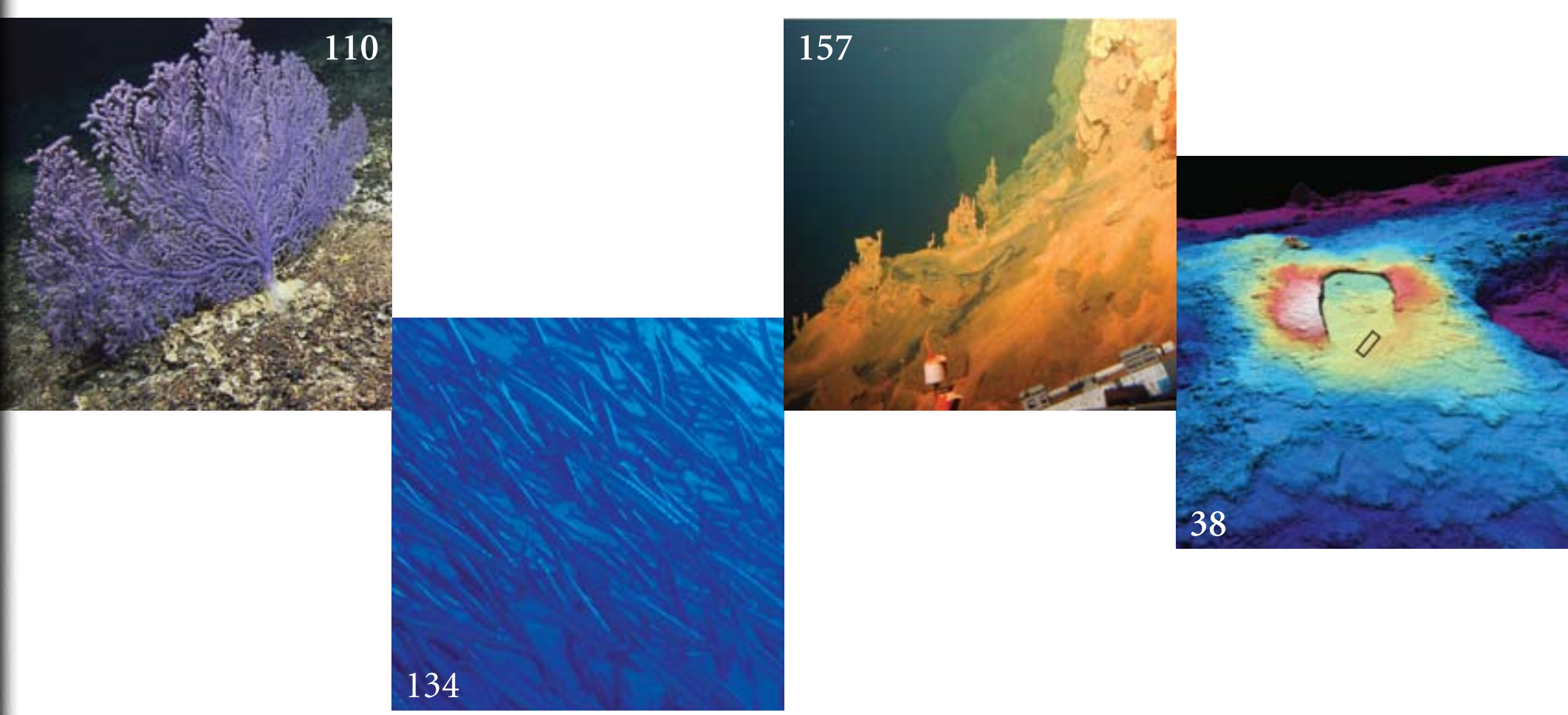

of "boxes" to explain concepts, new technologies, some special seamount research approaches, and the science underlying critical policies for conservation and management of seamounts and their biotic communities. At the end, a short synthesis sums up the highlights of seamount research presented in this issue, poses some of the next important questions for seamount research, and explores some of the most important steps to be taken to enhance seamount research in the near future. Lastly, we hope that this issue of Oceanography and continued seamount research will raise public awareness of the uniqueness of the seamount habitat, their increasingly appreciated role in the health of the ocean ecosystem, and hence their importance to humankind. This is a critical first step that will help formulate conservation policies that ideally will bring the seamount ecosystem back to a state of sustainability and health.

\section{ACKNOWLEDGEMENTS}

The Seamount Biogeoscience Network acknowledges the support by the US National Science Foundation, the (NSF-BIO) program for Research Coordination Networks and the NSF Ocean Sciences Directorate Marine Geology and Geophysics program (NSF-OCE), and CenSeam, the Census for Marine Life. This volume has benefited greatly from the hard work of the participants in Seamounts '09, in particular, the authors of the papers published in this issue and the efforts of the discussion group leaders Malcolm Clark, Al Hofmann, and Paul Wessel. We also wish to thank the large number of named and anonymous reviewers for their extraordinary diligence and constructive criticism, delivered under tight deadlines.

Hubert Staudigel, Scripps Institution of Oceanography

Anthony A.P. Koppers, Oregon State University

J. William Lavelle, National Oceanic and Atmospheric Administration

Tony J. Pitcher, University of British Columbia

Timothy M. Shank, Woods Hole Oceanographic Institution 